\title{
Antileishmanial Properties of Moroccan Medicinal Plants and Mechanism Insights of their Main Compounds
}

\author{
Abdelaali Balahbib ${ }^{1 \mathbb{D}}$, Nasreddine El Omari ${ }^{2}{ }^{\mathbb{D}}$, Abderrahim Sadak ${ }^{1}{ }^{\mathbb{D}}$, Youssef Bakri ${ }^{3}$, \\ Abdelhakim Bouyahya 3 ,* iD \\ ${ }^{1}$ Laboratory of Zoology and General Biology, Faculty of Sciences, Mohammed V University in Rabat, Morocco \\ ${ }^{2}$ Laboratory of Histology, Embryology, and Cytogenetic, Faculty of Medicine and Pharmacy, Mohammed V University in \\ Rabat, Morocco \\ ${ }^{3}$ Laboratory of Human Pathologies Biology, Department of Biology, Faculty of Sciences, and Genomic Center of Human \\ Pathologies, Faculty of Medicine and Pharmacy, Mohammed V University in Rabat, Morocco \\ * Correspondence: boyahyaa-90@hotmail.fr;
}

Scopus Author ID 57190813643

Received: 2.05.2020; Revised: 1.06.2020; Accepted: 2.06.2020; Published: 7.06.2020

\begin{abstract}
For several years, leishmaniasis was considered serious problem health of the Moroccan population. The used treatments against leishmaniasis are mostly expensive and present intolerable side effects. Furthermore, the search for antileishmanial bioactive compounds is urgent. Today, some studies started in Morocco, the evaluation of antileishmanial effects of natural products, in particular, bioactive compounds extracted from Moroccan medicinal plants. This work aims to explain the general epidemiological situation on the leishmaniasis in Morocco and to present all data about the antileishmanial properties of Moroccan medicinal plants. Several ethnopharmacological studies showed the use of Moroccan medicinal plants against leishmaniasis. The biological test showed that these plants showed cytotoxicity against Leishmania species, such as Leishmania major, Leishmania infantum, and Leishmania tropica. Moreover, mechanism insights of the main compound of tested medicinal plants showed specifically targeted pathways such as apoptosis action, topoisomerase inhibition, and respiratory chain perturbation.
\end{abstract}

Keywords: Leishmaniasis; Medicinal plants, Essential oils; Leishmanicidal effect.

(C) 2020 by the authors. This article is an open-access article distributed under the terms and conditions of the Creative Commons Attribution (CC BY) license (https://creativecommons.org/licenses/by/4.0/).

\section{Introduction}

Leishmaniasis is a parasitic vector disease caused by protozoa of the genus Leishmania. This disease has a significant impact in developing countries and a high mortality rate in Mediterranean areas, Africa, Latin America, and Asia [1]. This disease affects 12 million people in 98 countries [2,3]. The disease currently threatens about 350 million around the world. The current treatment by chemical drugs has several limitations such as toxic side effects, high cost, relapse, and the development of resistance [4,5], and requires long-term treatment [6]. Thus, an investment in drug development against parasitic diseases is a risky affair. In this context, the World Health Organization (WHO) has targeted research using plants to treat leishmaniasis [7]. Therefore, the research of new bioactive compounds with antileishmanial activity is necessary for the control and prevention of leishmaniasis [8]. In this context, certain studies reported that natural resources with effective antileishmanial activity might present a valuable source for the therapeutic control of leishmaniasis in humans. In Morocco, several works have shown the antileishmanial properties of natural resources [9]. In 
this review, we have reported all the Moroccan medicinal plants tested for antileishmanial effects, their traditional use, and the mechanisms of action involved.

\section{Overviews on Leishmaniasis}

Leishmaniasis is a parasitic disease of the monocyte-macrophage system whose pathogen is a flagellated protozoan of the genus Leishmania. This is a zoonosis transmitted from vertebrate to vertebrate by a hematophagous midge, the sandfly [10,11]. Only the female is hematophagous, his flight is silent and short-range, and his sting is painful but leaves no trace. It stings both man and animals, and she needs blood for the development of her eggs. Their lodging is constituted by the crevices of walls and burrows where they gorge themselves on micro-mammals (rodents, primates, marsupials, etc.) that may constitute the reservoir for some species of Leishmania.

Leishmaniasis includes visceral forms, localized cutaneous, diffuse cutaneous, and mucocutaneous forms. This multiplicity of clinical pictures results from a wide range of species and variation in the immune response of the infected host. The description of the first species of Leishmania was made by Laveran and Mesnil in 1903, and since, the number of species described has steadily increased. It is a parasite of vast geographical distribution found in Asia, Africa, South, and Central America and Europe, especially around the Mediterranean basin. Different species infect more than 15 million people, and there are two million new cases each year [12]. There are no effective vaccines yet to prevent the disease [13], and attempts to control the vector have so far been unsuccessful. This parasite presents during its cycle two distinct evolutionary stages; the amastigote stage, without flagella, is intramacrophagic and found in vertebrate hosts, including man and the promastigote stage, mobile thanks to its flagellum, is found in the intestine of sandflies and in culture media.

Its life cycle is indirect because parasite development occurs in two successive hosts; the definitive host is man, dog or animals (wild and domestic) and the intermediate host by the vector: the female sandfly. Leishmaniasis is transmitted to humans through the bite of infected female sandflies [10]. At the definitive host, the amastigote forms multiply in the cells of the reticuloendothelial system [14]. The host cell eventually explodes, releasing parasites that immediately enter new cells.

The sandfly, which is the intermediate host, gets infected by pricking a sick man or animal. It absorbs blood monocytes. In the digestive tract of the insect, the amastigote forms are transformed into promastigote forms. After about a week and following suction efforts, the sandfly injects the promastigote forms in the wound. The transformation into endocellular amastigote forms (in mononuclear phagocytes) is carried out in a few minutes. Sandflies inject the larva at the infectious stage, metacyclic promastigotes, during the blood meal. Metacyclic promastigotes that reach the wound are phagocytosed by the macrophages of the dermis and turn into amastigotes by losing their flagellum [10]. Amastigotes multiply and survive in phagolysosomes of infected cells. They can spread through the blood and lymphatic system and reach different tissues. The location of the parasite in the patient's various organs is directly related to the tropism of Leishmania species. Vector transmission is the main mode of contamination. There is also transmission by syringe exchange among drug addicts. Transfusion and congenital transmissions remain exceptional. 


\section{Leishmaniasis in Morocco}

Unfortunately, Morocco is one of the countries infected with leishmaniasis, and the disease is still major health problems posing a major threat to public health. Leishmaniases have been known in Morocco for nearly a century; the first cases of cutaneous leishmaniasis (CL) have been described since 1914 in the South [15]. Since the early 1970s, the situation of leishmaniasis has become worrying in Morocco [16]. The eco-epidemiological studies have revealed the simultaneous presence of three distinct noso-geographical forms that are differentiated by their geographical distribution, their clinical-epidemiological aspect, their causative agent as well as the vectors, and possible reservoirs [16].

After the launch of the National Program for the control of leishmaniasis carried out by the Department of Epidemiology and Disease, the epidemiological situation of leishmaniasis in all provinces and regions has enabled better monitoring [17]. A period of calm was noted from 1998 to 2001, during which the number of cases did not exceed 550 cases. The year 2008 was marked by a significant increase in cases to reach 3000 . This ascent continued to reach a peak of 6444 cases in 2010. These epidemics triggered the application of a national plan of response to stop this increase. The result of this plan resulted in a net decrease in cases in 2014 [18]. Unfortunately, from 2015 to 2016, a re-emergence of this form has been noticed in some households such as Jerada, Ouarzazate, Tinghir, and Errachidia [19].

The CL experienced a major epidemic south of the Anti-Atlas in the province of Tata. Subsequently, the disease spread to the provinces of Ouarzazate and Oujda [20]. Since the disease began to affect several other provinces with an epidemic character in the South and East of the country [21]. Nearly six provinces have been affected, namely the provinces of Errachidia, Figuig, Jerada, Ouarzazate, Boulemane, Tinghir, and Zagora [21,22].

L. tropica covers an area of 400 square kilometers [23]. Over the years, several other outbreaks have emerged, explaining the spatio-temporal extension of the disease in the country. In 2001, an epidemic was triggered in the provinces of Moulay Yacoub and Chichaoua [24]. The distribution of the cutaneous leishmaniasis from 2000 to 2015 has been maintained in an epidemic and constant manner in some provinces, such as Chichaoua, with an average of 342 cases/year $[19,25]$.

Cutaneous leishmaniasis, caused by L. infantum, has previously generated sporadic cases restricted to the provinces of Sidi Kacem and Taounate [21]. Molecular investigations have also revealed the presence of an increasing number of infected cases in the provinces of Ouazzane and Sidi Kacem [26,27]. Furthermore, visceral leishmaniasis (VL), also caused by $L$. infantum, is endemic in northern and central Morocco. This parasite mainly affects children under 5 years, with an estimated incidence of 140 cases per year [28].

Various protocols for the diagnosis of Leishmania have been used in Morocco, but the most used diagnosis is direct microscopy for searching for amastigote forms [29]. Sometimes, the confirmation of these cases is at the national reference laboratory using the molecular test, ITS1 PCR-RFLP method of the ribosomal DNA [30-32]. While other research applied the antibody detection methods such as immunochromatographic dipstick test (ICT), ELISA, IFAT with high sensitivity and specificity for ICT [33], and antigen detection assay test RDT for CL has also been developed [34]. Early diagnosis and prompt treatment may heal and prevent the development of stigmatizing lesions and scars [29]. 
In Morocco, entomological surveys showed the existence of specific vectors for each species. Effectively, Phlebotomus papatasi, Phlebotomus sergenti, and Phlebotomus longicuspis are the vectors of L. major [20], L. tropica [20], and L. infantum [35], respectively.

Table 1. Traditional use of Moroccan medicinal plants with antileishmanial activities.

\begin{tabular}{|c|c|c|c|}
\hline Species & $\begin{array}{l}\text { Vernacular } \\
\text { names }\end{array}$ & Used part & Medicinal indications \\
\hline $\begin{array}{l}\text { Origanum compactum } \\
\text { Benth. } \\
\text { Lamiaceae }\end{array}$ & Za'tar & $\begin{array}{l}\text { Flowering } \\
\text { top }\end{array}$ & $\begin{array}{ll}- & \text { Stomach disorders and febrifuge [38] } \\
\text { - } & \text { Inflammation [44] } \\
\text { - } & \text { Heart and intestinal pains [45] } \\
\text { - } & \text { Digestive problems, cardiovascular disorders, and } \\
& \text { respiratory problems [49] }\end{array}$ \\
\hline $\begin{array}{l}\text { Lavandula stoechas } \mathrm{L} . \\
\text { Lamiaceae }\end{array}$ & Halhal & $\begin{array}{l}\text { Flowering } \\
\text { top }\end{array}$ & $\begin{array}{ll}- & \text { Digestive disorders [38] } \\
\text { - } & \text { Rheumatism and asthma [44] } \\
\text { - } & \text { Rheumatism and digestive disorders [45] } \\
\text { - } & \text { Used as a diuretic [51] }\end{array}$ \\
\hline $\begin{array}{l}\text { Mentha pulegium } \mathrm{L} \text {. } \\
\text { Lamiaceae }\end{array}$ & Fliyou & $\begin{array}{l}\text { Flowering } \\
\text { top }\end{array}$ & $\begin{array}{ll}\text { - } & \text { Stomach disorders, cooling and respiratory diseases } \\
& \text { such as influenza, colds, and bronchitis [38] } \\
\text { - } & \text { Hypertension, diabetes, and cardiac disease [43] } \\
\text { - } & \text { Cold, respiratory canals [45] } \\
\text { - } & \text { Respiratory problems [49] } \\
\text { - } & \text { Cooling and flu [50] } \\
\text { - } & \text { Cough and chest problems [55] }\end{array}$ \\
\hline $\begin{array}{l}\text { Salvia officinalis L. } \\
\text { Lamiaceae }\end{array}$ & Salmia & Leaf & $\begin{array}{ll}- & \text { Hypoglycemia }[38,47] \\
- & \text { Hypertension and cardiac disease [43] } \\
- & \text { Asthma and inflammation [46] } \\
- & \text { Chill, rheumatism, and cough [45] } \\
- & \text { Diabetes [49] } \\
- & \text { Fever and stomach pains [50] } \\
\end{array}$ \\
\hline $\begin{array}{l}\text { Rosmarinus officinalis } \\
\text { L. } \\
\text { Lamiaceae }\end{array}$ & Azir & Leaf & $\begin{array}{ll}- & \text { Stomach disorders and cooling [38] } \\
- & \text { Hypertension, diabetes, and cardiac disease [43] } \\
\text { - } & \text { Allergy, asthma, cancer, infections, and immune system } \\
& \text { depression [44] } \\
\text { - } & \text { Intestinal parasites and rheumatism [45] } \\
- & \text { Diabetes [49] } \\
- & \text { Stomach pains [50] } \\
- & \text { Pyelonephritis and cystitis[51] } \\
\end{array}$ \\
\hline $\begin{array}{l}\text { Myrtus communis } \\
\text { Myrtaceae }\end{array}$ & Rihàn & Leaf & $\begin{array}{ll}- & \text { Fall protection [38] } \\
\text { - } & \text { Cardiac weakness and digestive system [41] } \\
- & \text { Immunological diseases [44] }\end{array}$ \\
\hline $\begin{array}{l}\text { Arbutus unedo L. } \\
\text { Ericaceae }\end{array}$ & Senou & Leaf & $\begin{array}{ll}- & \text { Diabetes [38] } \\
- & \text { Kidney diseases [45] }\end{array}$ \\
\hline $\begin{array}{l}\text { Cistus crispus } \mathrm{L} . \\
\text { Cistaceae }\end{array}$ & Ouekir & Leaf & - $\quad$ Skin wounds [38] \\
\hline $\begin{array}{l}\text { Centaurium } \\
\text { erythraea } \text { Rafn. } \\
\text { Gentianaceae }\end{array}$ & Korsatlhaya & $\begin{array}{l}\text { Flowering } \\
\text { top }\end{array}$ & $\begin{array}{ll}- & \text { Skin diseases [38] } \\
\text { - } & \text { Digestive system and kidney diseases [45] }\end{array}$ \\
\hline $\begin{array}{l}\text { Pistacia lentiscus } \mathrm{L} . \\
\text { Anacardiaceae }\end{array}$ & Drou & Leaf & - $\quad$ Digestive diseases and evil eye [45] \\
\hline $\begin{array}{l}\text { Salvia verbenaca } \\
\text { Lamiaceae }\end{array}$ & Hiyyata & Leaf & Wounds and abscesses [47] \\
\hline $\begin{array}{l}\text { Berberishispanica } \\
\text { Berberidaceae }\end{array}$ & Arris & Stem bark & - $\quad$ Cancer, Eczema, and Psoriasis [44] \\
\hline $\begin{array}{l}\text { Lavandula dentata } \\
\text { Lamiaceae }\end{array}$ & Khzama & Flowers & $\begin{array}{l}\text { - Hypertension, diabetes, and cardiac disease [42] } \\
\text { - } \\
\text { cough, gastrointestinal disorders, and neurological } \\
\text { conditions [50] }\end{array}$ \\
\hline $\begin{array}{l}\text { Cistus salviifolius } \\
\text { Cistaceae }\end{array}$ & Tarahla & Leaf & - $\quad$ Cutaneous leishmaniasis[53] \\
\hline $\begin{array}{l}\text { Crataegus oxyacantha } \\
\text { Rosaceae }\end{array}$ & Saarourchaik & Leaf & $\begin{array}{ll}- & \text { Hypertension and cardiac disease [42] }\end{array}$ \\
\hline $\begin{array}{l}\text { Ephedraaltissima } \\
\text { Ephedraceae }\end{array}$ & laâlenda & Tige & - $\quad$ Abortion [46] \\
\hline
\end{tabular}


Additionally, the Ministry of Health launched action plans, in partnership with other sectors, whose objectives were to identify $75 \%$ of the estimated cases of cutaneous leishmaniasis by 2021 , to eliminate mortality related to VL by 2021, and eliminate the disease in the country by 2030 [36].

\section{Medicinal use of Moroccan medicinal plants with antileishmanial effects}

In Morocco, the population still uses medicinal plants to treat a number of diseases such as diabetes, cancer, cardiovascular diseases, bacterial, and parasitic infections, including leishmaniasis. Indeed, several works carried out in different Moroccan regions and dating back to the '70s have reported the traditional use of medicinal plants [9, 37-58].

Amongst all used medicinal plants to fight against leishmaniasis, sixteen have been tested in Morocco against leishmaniasis; these plants are divided into 9 families (Lamiaceae, Myrtaceae, Ericaceae, Cistaceae, Gentianaceae, Anacardiaceae, Berberidaceae, Rosaceae, and Ephedraceae) (Table 1). The traditional use of these plants shows significant variability depending on the plant used, the region, and the pathology treated. Plants of the family Lamiaceae, namely (Origanum compactum Benth., Lavandula stoechas L., Mentha pulegium L., Salvia officinalis L., Rosmarinus officinalis L., Salvia verbenaca, Lavandula dentate) are the most used in traditional medicine. This use is certainly justified by the presence of high dose of secondary metabolite in these plants, especially because they express volatile substances or essential oils (EO) which have long shown enormous pharmacological properties, their use is often done by flowering tops and leaves, this is explained by the fact that these plants are the place of photosynthesis and therefore the presence of bioactive substances. Among these plants tested against leishmaniasis, the majority of them are traditionally used against leishmania. Indeed, Rosmarinus officinalis L., Berberis hispanica, Lavandula dentata, Cistus salviifolius, and Crataegus oxyacantha are used in the region of Fez [53] to fight cutaneous leishmania. In addition, several plants of the Lamiaceae family are also used against cutaneous Leishmania in the Tafilalt region [46].

\section{Antileishmanial activities of Moroccan medicinal plants}

Given the epidemiological situation of Leishmaniasis in Morocco and the resistance developed is aimed at drugs used, it is urgent to screen candidate molecules to alternatives of these medications. In this sense, medicinal plants are a reservoir of natural molecules with interesting biological activities. The secondary metabolites show interesting antileishmanial effects. However, in Morocco, the search for natural antileishmanial molecules has only recently been discussed in our laboratory. This activity was performed by organic extracts and volatile compounds.

\subsection{Antileishmanial activity of extracts.}

Several Moroccan medicinal plant extracts were tested against leishmania species (Table 2). These species include Myrtus communis, Salvia officinalis, Arbutus unedo, Origanum compactum, Cistus crispus, Centaurium erythraea, Berberis hispanica, Lavandula dentata, Cistus salviifolius, Crataegus oxyacantha, and Ephedra altissimoa (Table 2). Bouyahya et al. [62] have shown that organic extracts of Myrtus communis, Arbutus unedo, Origanum compactum, and Cistus crispus exhibited important antileishmanial activity. Indeed, methanol, hexan, and ethanol extract of Myrtus communis inhibited the growth of Leishmania 
infantum, Leishmania tropica, and Leishmania major. Moreover, Arbutus unedo methanolic, ethanolic, and hexane extracts showed remarkable leishmanicidal effects, in particularly hexane extract against Leishmania tropica $\left(\mathrm{IC}_{50}=79.57 \pm 2.66 \mu \mathrm{g} / \mathrm{mL}\right)$ and Leishmania infantum (IC50 $=64.05 \pm 1.44 \mu \mathrm{g} / \mathrm{mL}$ ). Cistus crispus extracts have also demonstrated interested in vitro antileishmanial effects, especially hexane extract that showed important leishmanicidal effects; Leishmania major $\left(\mathrm{IC}_{50}=47.29 \pm 2.25 \mu \mathrm{g} / \mathrm{mL}\right.$ ), Leishmania tropica $\left(\mathrm{IC}_{50}=96.82 \pm 2.88 \mu \mathrm{g} / \mathrm{mL}\right.$ ), and Leishmania infantum ( $\mathrm{IC}_{50}=82.39 \pm 2.11 \mu \mathrm{g} / \mathrm{mL}$ ). In another study, Bouyahya et al. [62] demonstrated Centaurium erythraea extracts against Leishmania tropica, L. major, and $L$. infantum. In this study, hexane extracts showed important antileishmanial activity against $L$. major $\left(\mathrm{IC}_{50}=64.52 \pm 2.2 \mu \mathrm{g} / \mathrm{mL}\right)$ and L. tropica $\left(\mathrm{IC}_{50}=37.2 \pm 1.62 \mu \mathrm{g} / \mathrm{mL}\right)[62]$.

Table 2. Antileishmanial effects of organic extracts isolated from Moroccan medicinal plants.

\begin{tabular}{|c|c|c|c|c|c|}
\hline Authors & Species & Used part & Extracts & Leishmania & Key results \\
\hline \multirow[t]{9}{*}{ [59] } & \multirow[t]{9}{*}{ Myrtus communis L. } & \multirow[t]{9}{*}{ Leaves } & \multirow[t]{3}{*}{ Methanol extract } & $\begin{array}{l}\text { L. major } \\
\text { promastigotes }\end{array}$ & Important inhibition \\
\hline & & & & $\begin{array}{l}L . \quad \text { tropica } \\
\text { promastigotes }\end{array}$ & $\mathrm{IC}_{50}=481.16 \pm 5.45 \mu \mathrm{g} / \mathrm{mL}$ \\
\hline & & & & $\begin{array}{l}L . \quad \text { infantum } \\
\text { promastigotes }\end{array}$ & $\mathrm{IC}_{50}=303.21 \pm 5.72 \mu \mathrm{g} / \mathrm{mL}$ \\
\hline & & & \multirow[t]{3}{*}{ Ethanol extract } & $\begin{array}{l}. \quad \text { major } \\
\text { promastigotes }\end{array}$ & Important inhibition \\
\hline & & & & $\begin{array}{l}L . \quad \text { tropica } \\
\text { promastigotes }\end{array}$ & Important inhibition \\
\hline & & & & $\begin{array}{l}L . \quad \text { infantum } \\
\text { promastigotes }\end{array}$ & $\mathrm{IC}_{50}=117.45 \pm 3.55 \mu \mathrm{g} / \mathrm{mL}$ \\
\hline & & & \multirow[t]{3}{*}{ Hexane extract } & $\begin{array}{l}L . \quad \text { major } \\
\text { promastigotes }\end{array}$ & $\mathrm{IC}_{50}=342.25 \pm 6.32 \mu \mathrm{g} / \mathrm{mL}$ \\
\hline & & & & $\begin{array}{l}L . \quad \text { tropica } \\
\text { promastigotes }\end{array}$ & $\mathrm{IC}_{50}=321.63 \pm 6.85 \mu \mathrm{g} / \mathrm{mL}$ \\
\hline & & & & $\begin{array}{l}L . \quad \text { infantum } \\
\text { promastigotes }\end{array}$ & Important inhibition \\
\hline \multirow[t]{9}{*}{ [60] } & \multirow[t]{9}{*}{ Salvia clandestina } & \multirow[t]{9}{*}{ Aerial part } & \multirow[t]{3}{*}{ Hexane extract } & $\begin{array}{lr}L . \quad \text { major } \\
\text { promastigotes }\end{array}$ & $\mathrm{IC}_{50}=155.43 \mu \mathrm{g} / \mathrm{mL}$ \\
\hline & & & & $\begin{array}{l}L . \quad \text { tropica } \\
\text { promastigotes }\end{array}$ & $\mathrm{IC}_{50}=148.23 \mu \mathrm{g} / \mathrm{mL}$ \\
\hline & & & & $\begin{array}{l}L . \quad \text { infantum } \\
\text { promastigotes }\end{array}$ & $\mathrm{IC}_{50}=14.11 \mu \mathrm{g} / \mathrm{mL}$ \\
\hline & & & \multirow[t]{3}{*}{$\begin{array}{l}\text { Dichloromethane } \\
\text { Extract }\end{array}$} & $\begin{array}{lr}L . \quad \text { major } \\
\text { promastigotes }\end{array}$ & $\mathrm{IC}_{50}=24.56 \mu \mathrm{g} / \mathrm{mL}$ \\
\hline & & & & $\begin{array}{lr}L . \quad \text { tropica } \\
\text { promastigotes }\end{array}$ & $\mathrm{IC}_{50}=33.77 \mu \mathrm{g} / \mathrm{mL}$ \\
\hline & & & & $\begin{array}{l}\text { L. infantum } \\
\text { promastigotes }\end{array}$ & $\mathrm{IC}_{50}=31.57 \mu \mathrm{g} / \mathrm{mL}$ \\
\hline & & & \multirow[t]{3}{*}{ Methanol extract } & $\begin{array}{l}L . \quad \text { major } \\
\text { promastigotes }\end{array}$ & $\mathrm{IC}_{50}>1000 \mu \mathrm{g} / \mathrm{mL}$ \\
\hline & & & & $\begin{array}{l}L . \quad \text { tropica } \\
\text { promastigotes }\end{array}$ & $\mathrm{IC}_{50}=850.76 \mu \mathrm{g} / \mathrm{mL}$ \\
\hline & & & & $\begin{array}{l}\text { L. infantum } \\
\text { promastigotes }\end{array}$ & $\mathrm{IC}_{50}>1000 \mu \mathrm{g} / \mathrm{mL}$ \\
\hline \multirow[t]{3}{*}{ [61] } & \multirow[t]{3}{*}{ Salvia officinalis L. } & \multirow[t]{3}{*}{ Leaves } & Hexane extract & $\begin{array}{lr}L . & \text { major } \\
\text { promastigotes }\end{array}$ & Important inhibition \\
\hline & & & Ethanol extract & $\begin{array}{lr}L . \quad \text { major } \\
\text { promastigotes }\end{array}$ & Important inhibition \\
\hline & & & Methanol extract & $\begin{array}{lr}L . & \text { major } \\
\text { promastigotes }\end{array}$ & Important inhibition \\
\hline \multirow[t]{4}{*}{ [59] } & \multirow[t]{4}{*}{ Arbutus unedo L. } & \multirow[t]{4}{*}{ Leaves } & \multirow[t]{3}{*}{ Methanol extract } & $\begin{array}{l}L . \quad \text { major } \\
\text { promastigotes }\end{array}$ & $\mathrm{IC}_{50}=283.83 \pm 4.96 \mu \mathrm{g} / \mathrm{mL}$ \\
\hline & & & & $\begin{array}{l}L . \quad \text { tropica } \\
\text { promastigotes }\end{array}$ & $\mathrm{IC}_{50}=103.74 \pm 3.22 \mu \mathrm{g} / \mathrm{mL}$ \\
\hline & & & & $\begin{array}{l}L . \quad \text { infantum } \\
\text { promastigotes }\end{array}$ & $\mathrm{IC}_{50}=150.23 \pm 3.21 \mu \mathrm{g} / \mathrm{mL}$ \\
\hline & & & Ethanol extract & $\begin{array}{lr}L . \quad \text { major } \\
\text { promastigotes }\end{array}$ & Important inhibition \\
\hline
\end{tabular}




\begin{tabular}{|c|c|c|c|c|c|}
\hline Authors & Species & Used part & Extracts & Leishmania & Key results \\
\hline & & & & $\begin{array}{l}L . \quad \text { tropica } \\
\text { promastigotes }\end{array}$ & Important inhibition \\
\hline & & & & $\begin{array}{l}\text { L. infantum } \\
\text { promastigotes }\end{array}$ & $\mathrm{IC}_{50}=172.72 \pm 3.56 \mu \mathrm{g} / \mathrm{mL}$ \\
\hline & & & Hexane extract & $\begin{array}{lr}L . \quad \text { major } \\
\text { promastigotes }\end{array}$ & $\mathrm{IC}_{50}=182.34 \pm 4.25 \mu \mathrm{g} / \mathrm{mL}$ \\
\hline & & & & $\begin{array}{l}\text { L. } \quad \text { tropica } \\
\text { promastigotes }\end{array}$ & $\mathrm{IC}_{50}=79.57 \pm 2.66 \mu \mathrm{g} / \mathrm{mL}$ \\
\hline & & & & $\begin{array}{l}L . \quad \text { infantum } \\
\text { promastigotes }\end{array}$ & $\mathrm{IC}_{50}=64.05 \pm 1.44 \mu \mathrm{g} / \mathrm{mL}$ \\
\hline \multirow[t]{9}{*}{ [59] } & \multirow[t]{9}{*}{$\begin{array}{l}\text { Origanum } \\
\text { compactum Benth. }\end{array}$} & \multirow[t]{9}{*}{$\begin{array}{l}\text { Flowering } \\
\text { stems }\end{array}$} & \multirow[t]{3}{*}{ Methanol extract } & $\begin{array}{l}L . \quad \text { major } \\
\text { promastigotes }\end{array}$ & Important inhibition \\
\hline & & & & $\begin{array}{l}\text { L. } \quad \text { tropica } \\
\text { promastigotes }\end{array}$ & Important inhibition \\
\hline & & & & $\begin{array}{l}\text { L. infantum } \\
\text { promastigotes }\end{array}$ & Important inhibition \\
\hline & & & \multirow[t]{3}{*}{ Ethanol extract } & $\begin{array}{lr}L . \quad \text { major } \\
\text { promastigotes }\end{array}$ & Important inhibition \\
\hline & & & & $\begin{array}{l}\text { L. tropica } \\
\text { promastigotes }\end{array}$ & Important inhibition \\
\hline & & & & $\begin{array}{l}L . \quad \text { infantum } \\
\text { promastigotes }\end{array}$ & $\mathrm{IC}_{50}=474.67 \pm 4.77 \mu \mathrm{g} / \mathrm{mL}$ \\
\hline & & & \multirow[t]{3}{*}{ Hexane extract } & $\begin{array}{l}L . \quad \text { major } \\
\text { promastigotes }\end{array}$ & $\mathrm{IC}_{50}=482.16 \pm 1.55 \mu \mathrm{g} / \mathrm{mL}$ \\
\hline & & & & $\begin{array}{l}\text { L. tropica } \\
\text { promastigotes }\end{array}$ & $\mathrm{IC}_{50}=289.68 \pm 4.15 \mu \mathrm{g} / \mathrm{mL}$ \\
\hline & & & & $\begin{array}{l}L . \quad \text { infantum } \\
\text { promastigotes }\end{array}$ & $\mathrm{IC}_{50}=275.94 \pm 5.76 \mu \mathrm{g} / \mathrm{mL}$ \\
\hline \multirow[t]{9}{*}{ [59] } & \multirow[t]{9}{*}{ Cistus crispus $\mathrm{L}$. } & \multirow[t]{9}{*}{ Leaves } & \multirow[t]{3}{*}{ Methanol extract } & $\begin{array}{lr}L . \quad \text { major } \\
\text { promastigotes }\end{array}$ & $\mathrm{IC}_{50}=84.29 \pm 2.05 \mu \mathrm{g} / \mathrm{mL}$ \\
\hline & & & & $\begin{array}{l}\text { L. tropica } \\
\text { promastigotes }\end{array}$ & $\mathrm{IC}_{50}=163.81 \pm 3.75 \mu \mathrm{g} / \mathrm{mL}$ \\
\hline & & & & $\begin{array}{l}L . \quad \text { infantum } \\
\text { promastigotes }\end{array}$ & $\mathrm{IC}_{50}=132.18 \pm 3.06 \mu \mathrm{g} / \mathrm{mL}$ \\
\hline & & & \multirow[t]{3}{*}{ Ethanol extract } & $\begin{array}{lr}L . \quad \text { major } \\
\text { promastigotes }\end{array}$ & $\mathrm{IC}_{50}=291.73 \pm 3.33 \mu \mathrm{g} / \mathrm{mL}$ \\
\hline & & & & $\begin{array}{l}\text { L. tropica } \\
\text { promastigotes }\end{array}$ & Important inhibition \\
\hline & & & & $\begin{array}{l}L . \quad \text { infantum } \\
\text { promastigotes }\end{array}$ & $\mathrm{IC}_{50}=183.26 \pm 4.38 \mu \mathrm{g} / \mathrm{mL}$ \\
\hline & & & \multirow[t]{3}{*}{ Hexane extract } & $\begin{array}{lr}L . \quad \text { major } \\
\text { promastigotes }\end{array}$ & $\mathrm{IC}_{50}=47.29 \pm 2.25 \mu \mathrm{g} / \mathrm{mL}$ \\
\hline & & & & $\begin{array}{l}L . \quad \text { tropica } \\
\text { promastigotes }\end{array}$ & $\mathrm{IC}_{50}=96.82 \pm 2.88 \mu \mathrm{g} / \mathrm{mL}$ \\
\hline & & & & $\begin{array}{l}L . \quad \text { infantum } \\
\text { promastigotes }\end{array}$ & $\mathrm{IC}_{50}=82.39 \pm 2.11 \mu \mathrm{g} / \mathrm{mL}$ \\
\hline \multirow[t]{9}{*}{ [62] } & \multirow[t]{9}{*}{$\begin{array}{l}\text { Centaurium } \\
\text { erythraea Rafin. }\end{array}$} & \multirow[t]{9}{*}{$\begin{array}{l}\text { Flowering } \\
\text { stems }\end{array}$} & \multirow[t]{3}{*}{ Methanol extract } & $\begin{array}{lr}L . \quad \text { major } \\
\text { promastigotes }\end{array}$ & $\mathrm{IC}_{50}=126.16 \pm 3.29 \mu \mathrm{g} / \mathrm{mL}$ \\
\hline & & & & $\begin{array}{l}\text { L. tropica } \\
\text { promastigotes }\end{array}$ & $\mathrm{IC}_{50}=247.24 \pm 2.59 \mu \mathrm{g} / \mathrm{mL}$ \\
\hline & & & & $\begin{array}{l}L . \quad \text { infantum } \\
\text { promastigotes }\end{array}$ & $\mathrm{IC}_{50}=124.82 \pm 1.75 \mu \mathrm{g} / \mathrm{mL}$ \\
\hline & & & \multirow[t]{3}{*}{ Ethanol extract } & $\begin{array}{lr}L . \quad \text { major } \\
\text { promastigotes }\end{array}$ & Important inhibition \\
\hline & & & & $\begin{array}{l}\text { L. } \quad \text { tropica } \\
\text { promastigotes }\end{array}$ & Important inhibition \\
\hline & & & & $\begin{array}{l}L . \quad \text { infantum } \\
\text { promastigotes }\end{array}$ & $\mathrm{IC}_{50}=373.18 \pm 2.23 \mu \mathrm{g} / \mathrm{mL}$ \\
\hline & & & \multirow[t]{3}{*}{ Hexane extract } & $\begin{array}{lr}L . \quad \text { major } \\
\text { promastigotes }\end{array}$ & $\mathrm{IC}_{50}=64.52 \pm 2.2 \mu \mathrm{g} / \mathrm{mL}$ \\
\hline & & & & $\begin{array}{l}\text { L. } \quad \text { tropica } \\
\text { promastigotes }\end{array}$ & $\mathrm{IC}_{50}=37.2 \pm 1.62 \mu \mathrm{g} / \mathrm{mL}$ \\
\hline & & & & $\begin{array}{l}L . \quad \text { infantum } \\
\text { promastigotes }\end{array}$ & $\mathrm{IC}_{50}=125.04 \pm 1.93 \mu \mathrm{g} / \mathrm{mL}$ \\
\hline \multirow[t]{2}{*}{ [53] } & \multirow[t]{2}{*}{ Berberis hispanica } & \multirow[t]{2}{*}{ Roots } & \multirow[t]{2}{*}{$\begin{array}{l}\text { Methanolic } \\
\text { extracts }\end{array}$} & $\begin{array}{l}\text { L. } \quad \text { tropica } \\
\text { promastigotes }\end{array}$ & $\mathrm{IC}_{50}=520.59 \pm 13.69 \mu \mathrm{g} / \mathrm{mL}$ \\
\hline & & & & $\begin{array}{lr}L . \quad \text { major } \\
\text { promastigotes }\end{array}$ & $\mathrm{IC}_{50}=526.41 \pm 4.50 \mu \mathrm{g} / \mathrm{mL}$ \\
\hline
\end{tabular}




\begin{tabular}{|c|c|c|c|c|c|}
\hline Authors & Species & Used part & Extracts & Leishmania & Key results \\
\hline & & & & $\begin{array}{l}L . \quad \text { infantum } \\
\text { promastigotse }\end{array}$ & $\mathrm{IC}_{50}=394.40 \pm 3.02 \mu \mathrm{g} / \mathrm{mL}$ \\
\hline & \multirow[t]{3}{*}{ Lavandula dentata } & \multirow[t]{3}{*}{ Leaves } & \multirow[t]{3}{*}{$\begin{array}{l}\text { Methanolic } \\
\text { extracts }\end{array}$} & $\begin{array}{l}\text { L. } \quad \text { tropica } \\
\text { promastigotes }\end{array}$ & $\mathrm{IC}_{50}=553.54 \pm 7.63 \mu \mathrm{g} / \mathrm{mL}$ \\
\hline & & & & $\begin{array}{l}L . \quad \text { major } \\
\text { promastigotes }\end{array}$ & $\mathrm{IC}_{50}=596.70 \pm 3.85 \mu \mathrm{g} / \mathrm{mL}$ \\
\hline & & & & $\begin{array}{l}L . \quad \text { infantum } \\
\text { promastigotes }\end{array}$ & $\mathrm{IC}_{50}=596.52 \pm 12.54 \mu \mathrm{g} / \mathrm{mL}$ \\
\hline & \multirow[t]{3}{*}{ Cistus salviifolius } & \multirow[t]{3}{*}{ Roots } & \multirow[t]{3}{*}{$\begin{array}{l}\text { Methanolic } \\
\text { extracts }\end{array}$} & $\begin{array}{l}\text { L. } \quad \text { tropica } \\
\text { promastigotes }\end{array}$ & $\mathrm{IC}_{50}=541.22 \pm 14.51 \mu \mathrm{g} / \mathrm{mL}$ \\
\hline & & & & $\begin{array}{l}L . \quad \text { major } \\
\text { promastigotes }\end{array}$ & $\mathrm{IC}_{50}=499.50 \pm 7.65 \mu \mathrm{g} / \mathrm{mL}$ \\
\hline & & & & $\begin{array}{l}L . \quad \text { infantum } \\
\text { promastigotes }\end{array}$ & $\mathrm{IC}_{50}=477.75 \pm 2.26 \mu \mathrm{g} / \mathrm{mL}$ \\
\hline & \multirow[t]{3}{*}{$\begin{array}{l}\text { Crataegus } \\
\text { oxyacantha }\end{array}$} & \multirow[t]{3}{*}{ Leaves } & \multirow[t]{3}{*}{$\begin{array}{l}\text { Methanolic } \\
\text { extracts }\end{array}$} & $\begin{array}{l}\text { L. } \quad \text { tropica } \\
\text { promastigotes }\end{array}$ & $\mathrm{IC}_{50}=546.31 \pm 7.73 \mu \mathrm{g} / \mathrm{mL}$ \\
\hline & & & & $\begin{array}{lr}L . \quad \text { major } \\
\text { promastigotes }\end{array}$ & $\mathrm{IC}_{50}=609.8 \pm 15.1 \mu \mathrm{g} / \mathrm{mL}$ \\
\hline & & & & $\begin{array}{l}L . \quad \text { infantum } \\
\text { promastigotes }\end{array}$ & $\mathrm{IC}_{50}=428.51 \pm 11.75 \mu \mathrm{g} / \mathrm{mL}$ \\
\hline & \multirow[t]{3}{*}{ Ephedra altissima } & \multirow[t]{3}{*}{ Roots } & \multirow[t]{3}{*}{$\begin{array}{l}\text { Methanolic } \\
\text { extracts }\end{array}$} & $\begin{array}{l}\text { L. } \quad \text { tropica } \\
\text { promastigotes }\end{array}$ & $\mathrm{IC}_{50}=540.11 \pm 12.49 \mu \mathrm{g} / \mathrm{mL}$ \\
\hline & & & & $\begin{array}{l}L . \quad \text { major } \\
\text { promastigotes }\end{array}$ & $\mathrm{IC}_{50}=490.84 \pm 3.15 \mu \mathrm{g} / \mathrm{mL}$ \\
\hline & & & & $\begin{array}{l}L . \quad \text { infantum } \\
\text { promastigotes }\end{array}$ & $\mathrm{IC}_{50}=535.22 \pm 4.39 \mu \mathrm{g} / \mathrm{mL}$ \\
\hline
\end{tabular}

\subsection{Antileishmanial activity of Essential oils.}

Volatile compounds of medicinal plants demonstrated remarkable anti-leishmanial activities. In Morocco, the anti-leishmanial activity of volatile compounds of some medicinal plants was evaluated. Table 3 summarizes all tested essential oils against Leishmania species. Essential oils of Origanum compactum, Pistacia lentiscus, Mentha pulegium, Rosmarinus officinalis, and Lavandula stoechas were tested against L. major, L. infantum, and L. tropica [63-66]. Volatile compounds extracted from flowering stems of Lavandula stoechas inhibited L. major ( $\mathrm{IC}_{50}=0.9 \pm 0.45 \mu \mathrm{g} / \mathrm{mL}$ ) and L. infantum $\left(\mathrm{IC}_{50}=7 \pm 0.54 \mu \mathrm{g} / \mathrm{mL}\right.$ importantly). This activity was attributed to Camphor and Fenchone, main major compounds of L. stoechas essential oil [66]. In another study, essential oils of Rosmarinus officinalis and Mentha pulegium showed important leishmanicidal action. Indeed, M. pulegium essential oil inhibited L. major at $\mathrm{IC}_{50}=1.3 \pm 0.45 \mu \mathrm{g} / \mathrm{mL}$ and $R$. officinalis essential oils inhibited Leishmania infantum at $\mathrm{IC}_{50}=1.2 \pm 0.36 \mu \mathrm{g} / \mathrm{mL}$ [65]. The variability between antileishmanial activities of different essential oils is certainly due to the difference in bioactive compounds containing in these oils. On the other, these volatile compounds could be explored to identified antileishmanial drugs in Morocco.

Table 3. Antileishmanial effects of essential oils isolated from Moroccan medicinal plants.

\begin{tabular}{|c|c|c|c|c|c|}
\hline Authors & Species & Used part & $\begin{array}{l}\text { Main } \\
\text { compounds }\end{array}$ & Leishmania & Key Results \\
\hline \multirow[t]{5}{*}{ [63] } & \multirow{5}{*}{$\begin{array}{l}\text { Origanum } \\
\text { compactum } \\
\text { Benth. }\end{array}$} & \multirow[t]{3}{*}{$\begin{array}{l}\text { Aerial part at } \\
\text { vegetative stage }\end{array}$} & \multirow{3}{*}{$\begin{array}{l}\text { Carvacrol, } \\
\text { thymol, } \\
\gamma \text {-terpenine, } \\
p \text {-cymene }\end{array}$} & $\begin{array}{lr}L . & \text { major } \\
\text { promastigotes }\end{array}$ & $\mathrm{IC}_{50}=0.26 \pm 0.09 \mu \mathrm{g} / \mathrm{mL}$ \\
\hline & & & & $\begin{array}{l}\text { L. infantum } \\
\text { promastigotes }\end{array}$ & $\mathrm{IC}_{50}=0.12 \pm 0.06 \mu \mathrm{g} / \mathrm{mL}$ \\
\hline & & & & $\begin{array}{l}\text { L. tropica } \\
\text { promastigotes }\end{array}$ & $\mathrm{IC}_{50}=0.35 \pm 0.03 \mu \mathrm{g} / \mathrm{mL}$ \\
\hline & & \multirow{2}{*}{$\begin{array}{l}\text { Flowering stems } \\
\text { at flowering } \\
\text { stage }\end{array}$} & \multirow{2}{*}{$\begin{array}{ll}\text { Carvacrol, } & \gamma- \\
\text { terpenine, } & \\
p \text {-cymene } & \end{array}$} & $\begin{array}{lr}L . \quad \text { major } \\
\text { promastigotes }\end{array}$ & $\mathrm{IC}_{50}=0.13 \pm 0.05 \mu \mathrm{g} / \mathrm{mL}$ \\
\hline & & & & $\begin{array}{l}L . \quad \text { infantum } \\
\text { promastigotes }\end{array}$ & $\mathrm{IC}_{50}=0.02 \pm 0.004 \mu \mathrm{g} / \mathrm{mL}$ \\
\hline
\end{tabular}




\begin{tabular}{|c|c|c|c|c|c|}
\hline \multirow[t]{5}{*}{ Authors } & \multirow[t]{5}{*}{ Species } & \multirow[t]{2}{*}{ Used part } & $\begin{array}{l}\text { Main } \\
\text { compounds }\end{array}$ & Leishmania & \multirow{2}{*}{$\begin{array}{l}\text { Key Results } \\
\mathrm{IC}_{50}=0.22 \pm 0.05 \mu \mathrm{g} / \mathrm{mL}\end{array}$} \\
\hline & & & & $\begin{array}{l}L . \quad \text { tropica } \\
\text { promastigotes }\end{array}$ & \\
\hline & & \multirow{3}{*}{$\begin{array}{l}\text { Aerial part at } \\
\text { post-flowering } \\
\text { stage }\end{array}$} & \multirow{3}{*}{$\begin{array}{l}\text { Thymol, } \\
\text { terpenine, } \\
p \text {-cymene }\end{array}$} & $\begin{array}{l}L . \quad \text { major } \\
\text { promastigotes }\end{array}$ & $\mathrm{IC}_{50}=0.17 \pm 0.02 \mu \mathrm{g} / \mathrm{mL}$ \\
\hline & & & & $\begin{array}{l}L . \quad \text { infantum } \\
\text { promastigotes }\end{array}$ & $\mathrm{IC}_{50}=0.04 \pm 0.01 \mu \mathrm{g} / \mathrm{mL}$ \\
\hline & & & & $\begin{array}{l}\text { L. tropica } \\
\text { promastigotes }\end{array}$ & $\mathrm{IC}_{50}=0.72 \pm 0.02 \mu \mathrm{g} / \mathrm{mL}$ \\
\hline \multirow[t]{6}{*}{ [64] } & \multirow[t]{6}{*}{$\begin{array}{l}\text { Pistacia lentiscus } \\
\text { L. }\end{array}$} & \multirow[t]{3}{*}{ Leaves } & \multirow[t]{3}{*}{$\begin{array}{l}\text { Myrcene } \\
\alpha \text {-pinene }\end{array}$} & $\begin{array}{l}L . \quad \text { major } \\
\text { promastigotes }\end{array}$ & $\mathrm{IC}_{50}=17.52 \pm 1.26 \mu \mathrm{g} / \mathrm{mL}$ \\
\hline & & & & $\begin{array}{l}L . \quad \text { infantum } \\
\text { promastigotes }\end{array}$ & $\mathrm{IC}_{50}=11.28 \pm 1.63 \mu \mathrm{g} / \mathrm{mL}$ \\
\hline & & & & $\begin{array}{l}\text { L. tropica } \\
\text { promastigotes }\end{array}$ & $\mathrm{IC}_{50}=23.5 \pm 3.38 \mu \mathrm{g} / \mathrm{mL}$ \\
\hline & & \multirow[t]{3}{*}{ Fruits } & \multirow[t]{3}{*}{$\begin{array}{l}\text { Limonene } \\
\alpha \text {-pinene }\end{array}$} & $\begin{array}{lr}L . \quad \text { major } \\
\text { promastigotes }\end{array}$ & $\mathrm{IC}_{50}=21.42 \pm 2.92 \mu \mathrm{g} / \mathrm{mL}$ \\
\hline & & & & $\begin{array}{l}L . \quad \text { infantum } \\
\text { promastigotes }\end{array}$ & $\mathrm{IC}_{50}=08 \pm 0.83 \mu \mathrm{g} / \mathrm{mL}$ \\
\hline & & & & $\begin{array}{l}L . \quad \text { tropica } \\
\text { promastigotes }\end{array}$ & $\mathrm{IC}_{50}=26.2 \pm 3.54 \mu \mathrm{g} / \mathrm{mL}$ \\
\hline \multirow[t]{6}{*}{ [65] } & \multirow[t]{3}{*}{$\begin{array}{l}\text { Mentha pulegium } \\
\text { L. }\end{array}$} & \multirow[t]{3}{*}{ Leaves } & \multirow[t]{3}{*}{$\begin{array}{l}\text { Menthone } \\
\text { Pulegone }\end{array}$} & $\begin{array}{l}L . \quad \text { major } \\
\text { promastigotes }\end{array}$ & $\mathrm{IC}_{50}=1.3 \pm 0.45 \mu \mathrm{g} / \mathrm{mL}$ \\
\hline & & & & $\begin{array}{l}L . \quad \text { infantum } \\
\text { promastigotes }\end{array}$ & $\mathrm{IC}_{50}=2 \pm 0.83 \mu \mathrm{g} / \mathrm{mL}$ \\
\hline & & & & $\begin{array}{l}\text { L. tropica } \\
\text { promastigotes }\end{array}$ & $\mathrm{IC}_{50}=2.2 \pm 0.25 \mu \mathrm{g} / \mathrm{mL}$ \\
\hline & \multirow[t]{3}{*}{$\begin{array}{l}\text { Rosmarinus } \\
\text { officinalis } \mathrm{L} \text {. }\end{array}$} & \multirow[t]{3}{*}{ Leaves } & \multirow{3}{*}{$\begin{array}{l}\alpha \text {-pinene } \\
1,8 \text {-Cineole } \\
\text { Borneol }\end{array}$} & $\begin{array}{lr}L . & \text { major } \\
\text { promastigotes }\end{array}$ & $\mathrm{IC}_{50}=2.6 \pm 0.64 \mu \mathrm{g} / \mathrm{mL}$ \\
\hline & & & & $\begin{array}{l}L . \quad \text { infantum } \\
\text { promastigotes }\end{array}$ & $\mathrm{IC}_{50}=1.2 \pm 0.36 \mu \mathrm{g} / \mathrm{mL}$ \\
\hline & & & & $\begin{array}{l}\text { L. tropica } \\
\text { promastigotes }\end{array}$ & $\mathrm{IC}_{50}=3.5 \pm 0.83 \mu \mathrm{g} / \mathrm{mL}$ \\
\hline \multirow[t]{3}{*}{ [66] } & \multirow[t]{3}{*}{$\begin{array}{l}\text { Lavandula } \\
\text { stoechas } \mathrm{L} .\end{array}$} & \multirow[t]{3}{*}{ Flowering stems } & \multirow[t]{3}{*}{$\begin{array}{l}\text { Camphor } \\
\text { Fenchone }\end{array}$} & $\begin{array}{lr}L . \quad \text { major } \\
\text { promastigotes }\end{array}$ & $\mathrm{IC}_{50}=0.9 \pm 0.45 \mu \mathrm{g} / \mathrm{mL}$ \\
\hline & & & & $\begin{array}{l}L . \quad \text { infantum } \\
\text { promastigotes }\end{array}$ & $\mathrm{IC}_{50}=7 \pm 0.54 \mu \mathrm{g} / \mathrm{mL}$ \\
\hline & & & & $\begin{array}{l}L . \quad \text { tropica } \\
\text { promastigotes }\end{array}$ & Important inhibition \\
\hline
\end{tabular}

\section{Leishmanicidal mechanism insights of Medicinal plants bioactive compounds}

Since then, the pharmaceutical industry has implemented antileishmanial molecules such as amphotericin B and pentamidine for L. brasiliensis [67]. Amarogentin for L. donovani [68] and Anophonin for L. major [69]. During this time, these molecules have shown undesirable and toxic effects, and they have many disadvantages [70]. For this reason, the screening of new natural molecules such as from medicinal plants is an effective and possible approach, a new hope of treating this much-neglected disease at the moment. In Morocco, a number of bioactive molecules present in antileishmanial medicinal plants have revealed leishmanicidal effects. Several studies (Table 4) have described the activity effects of bioactive molecules against leishmania.

Table 4. Leishmanicidal mechanism insights of Medicinal plants bioactive compounds.

\begin{tabular}{l|l|l|l|l|} 
Molecule & Species & Effects & Reference & Chemical structure \\
\hline \multirow{2}{*}{ Limonene } & $\begin{array}{l}\text { Leishmania amazonensis } \\
\text { promastigotes }\end{array}$ & $\mathrm{IC}_{50}=252.0 \pm 49.0 \mathrm{mM}$ & {$[71]$} \\
\cline { 2 - 4 } & $\begin{array}{l}\text { Leishmania amazonensis } \\
\text { amastigotes }\end{array}$ & $\mathrm{IC}_{50}=147.0 \pm 46.0 \mathrm{mM}$ & {$[71]$} \\
\cline { 2 - 4 } & $\begin{array}{l}\text { Leishmania major } \\
\text { promastigotes }\end{array}$ & $\mathrm{IC}_{50}=354.0 \pm 33.0 \mathrm{mM}$ & {$[71]$} \\
\hline
\end{tabular}




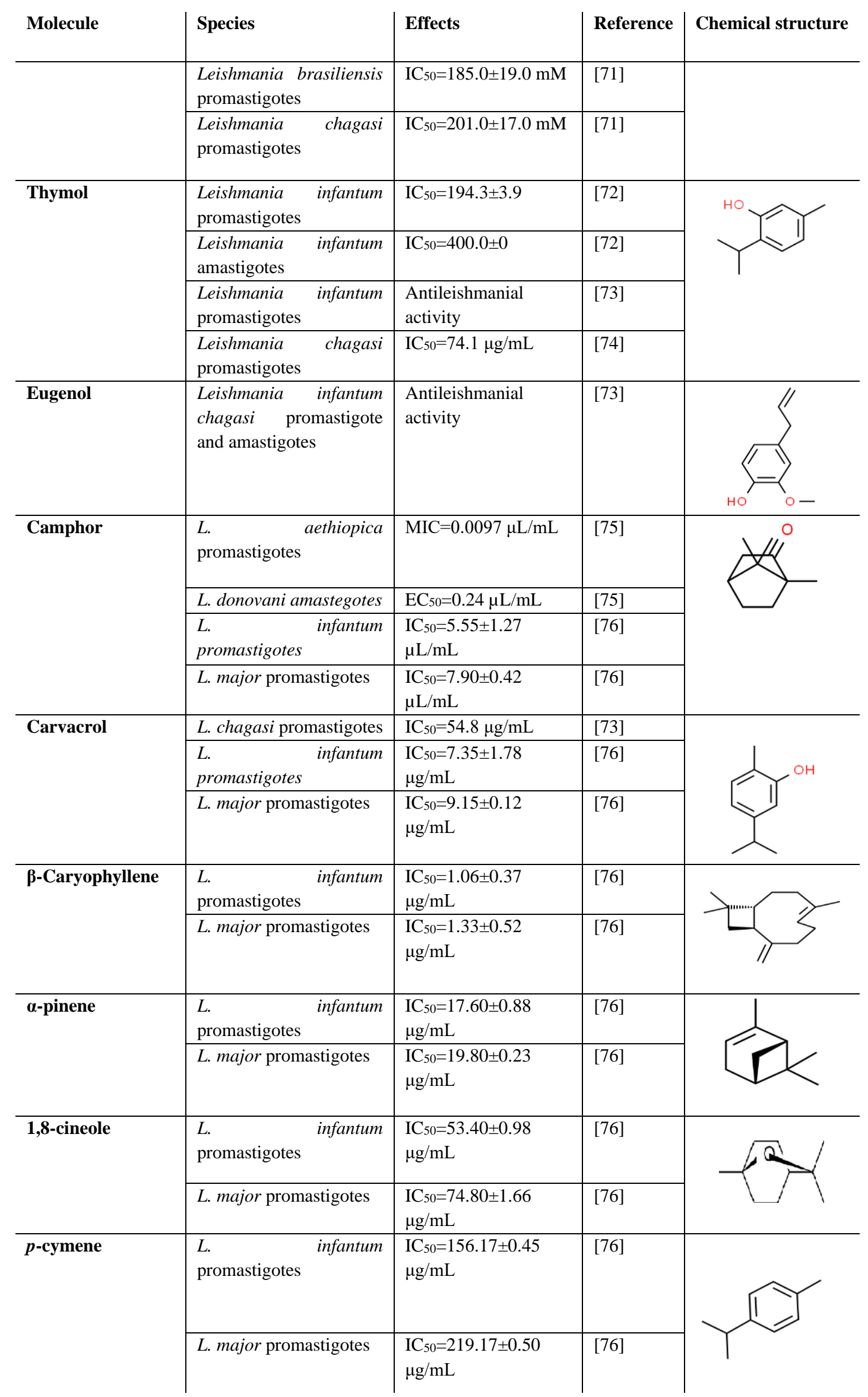

Arruda et al. (2009) demonstrated the activity of limonene against Leishmania species in vitro and in vivo. This molecule has a leishmanicidal action on L. amazonensis promastigotes and amastigotes, and was also effective against L. major, L. braziliensis, and L. chagasi 
promastigotes. In addition, the treatment with limonene allowed the reduction of the lesion and a decrease in the parasite load [71]. Additionally, several studies tested the antileishmanial activity for thymol in vitro, and in vivo, they show that this molecule is active against the parasite. It decreased the parasite burden for L. panamensis [72], and it is the best inhibitor and exhibited efficient leishmanicidal activity against L. infantum [73,74]. Another study shows that eugenol possesses a better antileishmanial activity against promastigotes the L. infantum and L. chagasi.

Moreover, the thymol has greater activity than the eugenol derivatives [73]. In another work, the results revealed that camphor exhibited an important leishmanicidal activity against amastigote forms of $L$. aethiopica with potent leishmanicidal activity against promastigotes of L. donovani [75], it may still be an alternative treatment in the therapy of leishmaniasis because of its activity against L. infantum and L. major [76]. Otherwise, carvacrol exhibited efficient leishmanicidal activity on promastigotes of L. chagasi [74]. L. brasiliensis [77], L. infantum and L. major [76], and its mechanism of action might involve alteration of the mitochondrial membrane [76]. Essid et al. [76] demonstrated that p-cymene, 1,8-cineole, $\alpha$-pinene, and caryophyllene exhibited efficient leishmanicidal activity against a different type of Leishmania, mostly on promastigotes of L. major and L. infantum [76]. Moreover, these molecules show the different modes of action, such as inhibition of proliferation, inhibition of Leishmania growth and mediation of apoptosis-like cell death, loss of mitochondrial membrane potential, and cell-cycle arrest [78]. These results show the importance of the investigation of molecules with therapeutic potential in the treatment of leishmaniasis.

\section{Conclusions and perspectives}

Leishmaniasis constitutes a dangerous parasitic disease that affects several African countries, including Morocco. To fight against this microbial disease, the Moroccan population uses medicinal plants as alternative treatments. Indeed, the used antileishmanial antibiotics often have several side effects and are expensive. The tested Moroccan medicinal plants against different species of Leishmania showed that the bioactive compounds contained in these plants could be a valuable source for identifying new alternative drugs against leishmaniasis. However, further investigations regarding the isolation of these compounds and their preclinical in vivo exploration and eventually, clinical validation are necessary.

\section{Funding}

This research received no external funding.

\section{Acknowledgments}

This research has no acknowledgment.

\section{Conflicts of Interest}

The authors declare no conflict of interest.

\section{References}

1. Rocha, L.G.; Almeida, J.R.G.S.; Macedo, R.O.; Barbosa-Filho, J.M. A review of natural products with antileishmanial activity. Phytomedicine 2005, 12, 514-535, https://doi.org/10.1016/j.phymed.2003.10.006. 
2. Alvar, J.; Vélez, I.D.; Bern, C.; Herrero, M.; Desjeux, P.; Cano, J. WHO Leishmaniasis Control Team. Leishmaniasis worldwide and global estimates of its incidence. PloS one 2012, 7, https://doi.org/10.1371/journal.pone.0035671.

3. Gomes, M.N.; Alcântara, L.M.; Neves, B.J.; Melo-Filho, C.C.; Freitas-Junior, L.H.; Moraes, C.B.; Ma, R.; Franzblau, S.G.; Muratov, E.; Andrade, C.H. Computer-aided discovery of two novel chalcone-like compounds active and selective against Leishmania infantum. Bioorganic \& medicinal chemistry letters 2017, 27, 2459-2464, file://Users/macbookair/Desktop/gomes2017\%20(1).pdf.

4. Oliveira, L.F.; Schubach, A.O.; Martins, M.M.; Passos, S.L.; Oliveira, R.V.; Marzochi, M.C.; Andrade, C.A. Systematic review of the adverse effects of cutaneous leishmaniasis treatment in the New World. Acta tropical 2011, 118, 87-96, https://doi.org/10.1016/j.actatropica.2011.02.007.

5. Murugan, N.; Natarajan, D. Bionanomedicine for antimicrobial therapy - a case study from Glycosmis pentaphylla plant mediated silver nanoparticles for control of multidrug resistant bacteria. Letters in Applied NanoBioScience 2018, 8, 523-540.

6. Haldar, A.K.; Sen, P.; Roy, S. Use of antimony in the treatment of leishmaniasis: current status and future directions. Molecular biology international 2011.

7. World Health Organization. Report of a meeting of the WHO Expert Committee on the Control of Leishmaniases, Geneva, Switzerland, 22-26 March 2010. WHO Technical Report Series 2010, 949.

8. Minodier, P.; Parola, P. Cutaneous leishmaniasis treatment. Travel medicine and infectious disease 2007, 5, 150-158, https://doi.org/10.1016/j.tmaid.2006.09.004.

9. Et-Touys, A.; Bouyahyal, A.; Fellah, H.; Mniouil, M.; El Bouryl, H.; Dakka, N.; Bakri, Y. Antileishmanial activity of medicinal plants from Africa: A review. Asian Pacific Journal of Tropical Disease 2017, 7, 826840, https://doi.org/10.12980/apjtd.7.2017D7-215.

10. Santos, D.O.; Coutinho, C.E.; Madeira, M.F.; Bottino, C.G.; Vieira, R.T.; Nascimento, S.B.; Rodrigues, C.R. Leishmaniasis treatment a challenge that remains: a review. Parasitology research 2008, 103, 1-10, https://doi.org/10.1007/s00436-008-0943-2.

11. Grimaldi Jr, G.; Tesh, R.B.; McMahon-Pratt, D. A review of the geographic distribution and epidemiology of leishmaniasis in the New World. The American journal of tropical medicine and hygiene 1989, 41, 687725, https://doi.org/10.4269/ajtmh.1989.41.687.

12. Herwaldt, B.L. Leishmaniasis. Lancet 1999, 354, 1191-9, https://doi.org/10.1016/s0140-6736(98)10178-2.

13. Ahmadian, S.; Orlov, Y.L.; Maurady, A.; Eslami, G.; Hosseini, S.S. RNA Polymrase II gene expression in clinical Leishmania major isolates with no-response-to-drug pattern. Biointerface Research in Applied Chemistry 2019, 9, 4126-4130, https://doi.org/10.33263/BRIAC94.126130.

14. Gangneux, J.P.; Robert-Gangneux, F. Les infections parasitaires du système des phagocytes mononucléés. Revue Francophone des Laboratoires 2006, 2006, 57-68, https://doi.org/10.1016/S1773-035X(06)80538-1.

15. Foley, H.; Vialatte, C. Existence dans le Sud marocain du bouton d'orient à l'état endémique. Bull. Soc. Path. Exot 1914, 7, 114-5.

16. Chaara, D.; Haouas, N.; Dedet, J.P.; Babba, H.; Pratlong, F. Leishmaniases in Maghreb: an endemic neglected disease. Acta tropica 2014, 132, 80-93, https://doi.org/10.1016/j.actatropica.2013.12.018.

17. Ministère de la Santé, La Direction de l'Epidémiologie et la Lutte contre les Maladies. Eco-Epidémiologie des leishmanioses au Maroc. Bulletin épidémiologique 1993, 37, 1-15

18. Ministère de la santé, Direction de l'Epidémiologie et de Lutte contre les Maladies (DELM). Etat d'avancement des programmes de lutte contre les maladies parasitaires, Rabat-Maroc, 2014.

19. Ministère de la santé, Direction de l'Epidémiologie et de Lutte contre les Maladies (DELM). Etat d'avancement des programmes de lutte contre les maladies parasitaires, Rabat-Maroc, 2015.

20. Rioux, J.A.; Daoud, W.; Pratlong, F.; El Kubati, Y.; Moreno, G.; Rageh, H.A.; Brun, R.; Mouharen, A.; Martinez Ortega, E.; Belmonte, A. Les complexes Leishmania donovani s. st., Leishmania tropica et Leishmania major en république arabe du yémen. Considérations taxonomiques et épidémiologiques. In: Rioux, J.A. (Ed.), Leishmania Taxonomie et phylogenèse. Applications éco-épidémiologiques. IMEEE, Montpellier, France 1986, 357-363.

21. Rhajaoui, M.; Nasereddin, A.; Fellah, H.; Azmi, K.; Amarir, F.; Al-Jawabreh, A.; Abdeen, Z. New clinicoepidemiologic profile of cutaneous leishmaniasis, Morocco. Emerging infectious diseases 2007, 13, 1358-1360.

22. Riyad, M.; Chiheb, S.; Soussi A.M. Cutaneous leishmaniasis caused by Leishmania major in Morocco: still a topical question. WHO 2013.

23. Pratlong, F.; Rioux, J. A.; Dereure, J.; Mahjour, J.; Gallego, M.; Guilvard, E.; Saddiki, A. Leishmania tropica in Morocco. IV--Intrafocal enzyme diversity. Annales de parasitologie humaine et comparee 1991, 66, 100104, https://doi.org/10.1051/parasite/1991663100.

24. DELM. Etat d'avancement des programmes de lutte contre les maladies parasitaires. Direction épidémiologique de lutte contre les maladies. Rabat, Maroc 2001.

25. Rhajaoui, M.; Sebti, F.; Fellah, H.; Alam, M.Z.; Nasereddin, A.; Abbasi, I.; Schönian, G. Identification of the causative agent of cutaneous leishmaniasis in Chichaoua province, Morocco. Parasite: journal de la Société Française de Parasitologie 2012, 19, 81-4, https://doi.org/10.1051/parasite/2012191081. 
26. El Miri, H.; Faraj, C.; Himmi, O.; Hmamouch, A.; Maniar, S.; Laaroussi, T.; Benhoussa, A. Cutaneous leishmaniasis in Ouazzane and Sidi Kacem provinces, Morocco (1997-2012). Bulletin de la Société de pathologie exotique 2016, 109, 376-380, https://doi.org/10.1007/s13149-016-0522-1.

27. El Aasri, A.; El Madhi, Y.; Najy, M.; El Rhaouat, O.; Belghyti, D. Epidemiology of cutaneous leishmaniasis in Sidi Kacem province, northwestern Morocco (2006-2014). Asian Pacific Journal of Tropical Disease 2016, 6, 783-786.

28. Mniouil, M.; Fellah, H.; Amarir, F.; Et-touys, A.; Bekhti, K.; Adlaoui, E.B.; Sebti, F. Epidemiological characteristics of visceral leishmaniasis in Morocco (1990-2014): an update. Acta tropica 2017, 170, 169177, https://doi.org/10.1016/j.actatropica.2016.10.016.

29. de Vries, H.J.; Reedijk, S.H.; Schallig, H.D. Cutaneous leishmaniasis: recent developments in diagnosis and management. American journal of clinical dermatology 2015, 16, 99-109, https://doi.org/10.1007/s40257015-0114-z.

30. Mahmoud, M.; Alem, E.; Faiza, S.; Lemine, M.; Smaine, C.; Adlaoui, E.B.; Hajiba, F. Geographical distribution and new situation of leishmania species after the control of cutaneous leishmaniasis foci in errachidia province, Morocco, in 2014. BioMed research international 2016.

31. Hakkour, M.; Hmamouch, A.; El Alem, M.M.; Rhalem, A.; Amarir, F.; Touzani, M.; Sebti, F. New epidemiological aspects of visceral and cutaneous leishmaniasis in Taza, Morocco. Parasites \&vectors 2016, 9,612 .

32. Hmamouch, A.; El Alem, M.M.; Hakkour, M.; Amarir, F.; Daghbach, H.; Habbari, K.; Sebti, F. Circulating species of Leishmania at microclimate area of Boulemane Province, Morocco: impact of environmental and human factors. Parasites \&vectors 2017, 10, 100, https://doi.org/10.1186/s13071-017-2032-9.

33. Mniouil, M.; Fellah, H.; Amarir, F.; Sadak; A.; Et-Touys; A., Bakri, Y.; Adlaoui, E.B. Comparative evaluation of immunochromatographic dipstick test (ICT) rk39, soluble antigen ELISA and IFAT for the sero-diagnosis of visceral leishmaniasis in Morocco. Acta tropica 2018, 182, 185-189, https://doi.org/10.1016/j.actatropica.2018.03.007.

34. Bennis, I.; De Brouwere, V.; Ameur, B.; El Idrissi Laamrani, A.; Chichaoui, S.; Hamid, S.; Boelaert, M. Control of cutaneous leishmaniasis caused by Leishmania major in south-eastern Morocco. Tropical Medicine \& International Health 2015, 20, 1297-1305, https://doi.org/10.1111/tmi.12543.

35. El Miri, H.; Rhajaoui, M.; Himmi, O.; Ouahabi, S.; Benhoussa, A.; Faraj, C. Etude entomologique de cinq foyers de leishmaniose cutanée dans la province de Sidi Kacem au nord du Maroc. Annales de la Société entomologique de France. Taylor \& Francis 2013, 49, 154-159, https://doi.org/10.1080/00379271.2013.808504.

36. Moroccan Ministry of Health. DELM. Bulletin of epidemiology and health in Morocco, 2018.

37. Slimani, I.; Nassiri, L.; Boukil, A.; Bouiamrine, E.H.; Bachiri, L.; Bammou, M.; Ibijbijen, J. Inventaire des plantes aromatiques et médicinales du site d'intérêt biologique et écologique de Jbel Zerhoun, région Meknès Tafilalet. Afrique Science 2016, 12, 393-409.

38. Bouyahya, A.; Abrini, J.; Et-Touys, A.; Bakri, Y.; Dakka, N. Indigenous knowledge of the use of medicinal plants in the North-West of Morocco and their biological activities. European Journal of Integrative Medicine 2017, 13, 9-25, https://doi.org/10.1016/j.eujim.2017.06.004.

39. Baghiani, A.; Boumerfeg, S.; Adjadj, M.; Ameni, D.; Djermouni, M.; Khelifi-Touhami, F.; Arrar, L. Antioxidants, Free Radicals Scavenging and Xanthine Oxidase Inhibitory Potentials of Ajuga iva L. Extracts. Free Radicals and Antioxidants 2011, 1, 21-30, https://doi.org/10.5530/ax.2011.4.5.

40. Bammi, J.; Mouhiddine, M.; Fassi, D.; Douira, A. Contribution à la connaissance de la végétation des Doukkala-Abda (Maroc Atlantique): Approche éco-géomorphologique. J. Anim. Plant Sci 2014, 20, 32023211.

41. Bellakhdar, J.; Claisse, R.; Fleurentin, J.; Younos, C. Repertory of standard herbal drugs in the Moroccan pharmacopoea. Journal of Ethnopharmacology 1991, 35, 123-143, https://doi.org/10.1016/03788741(91)90064-k.

42. Bnouham, M.; Mekhfi, H.; Legssyer, A.; Ziyyat, A. Ethnopharmacology Forum Medicinal plants used in the treatment of diabetes in Morocco. Int J Diabetes \& Metabolism 2002, 10, 33-50.

43. Eddouks, M.; Maghrani, M.; Lemhadri, A.; Ouahidi, M.L.; Jouad, H. Ethnopharmacological survey of medicinal plants used for the treatment of diabetes mellitus, hypertension and cardiac diseases in the southeast region of Morocco (Tafilalet). Journal of Ethnopharmacology 2002, 82, 97-103, https://doi.org/10.1016/S0378-8741(02)00164-2.

44. Youbi, A.E.H.E.; Ouahidi, I.; Mansouri, L.E.; Daoudi, A.; Bousta, D. Ethnopharmacological survey of plants used for immunological diseases in four regions of Morocco. European Journal of Medicinal Plants 2016, $13,1$.

45. El-Hilaly, J.; Hmammouchi, M.; Lyoussi, B. Ethnobotanical studies and economic evaluation of medicinal plants in Taounate province (Northern Morocco). Journal of Ethnopharmacology 2002, 86, 149-158, https://doi.org/10.1016/S0378-8741(03)00012-6.

46. El Rhaffari, L.; Zaid, A. Pratique de la phytothérapie dans le sud-est du Maroc (Tafilalet): Un savoir empirique pour une pharmacopée rénovée. $\mathbf{2 0 0 2}$. 
47. Benlamdini, N.; Elhafian, M.; Rochdi, A.; Zidane, L. Étude floristique et ethnobotanique de la flore médicinale du Haut Atlas oriental (Haute Moulouya). Journal of Applied Biosciences 2014, 78, 6771-6787, https://doi.org/10.4314/jab.v78i1.17.

48. Cotonou, B. Étude ethnobotanique des plantes utilisées dans le traitement du diabète chez les femmes enceintes à Cotonou et Abomey-Calavi (Bénin). Journal of Animal \& Plant Sciences 2013, 18, 2647-2658.

49. Jamila, F.; Mostafa, E. Ethnobotanical survey of medicinal plants used by people in Oriental Morocco to manage various ailments. Journal of ethnopharmacology 2014, 154, 76-87.

50. El Hafian, M.; Benlandini, N.; Elyacoubi, H.; Zidane, L.; Rochdi, A. Étude floristique et ethnobotanique des plantes médicinales utilisées au niveau de la préfecture d'Agadir-Ida-Outanane (Maroc). Journal of Applied Biosciences 2014, 81, 7198-7213, https://doi.org/10.4314/jab.v81i1.8.

51. Ghourri, M.; Zidane, L.; Douira, A. La phytothérapie et les infections urinaires (La pyélonéphrite et la cystite) au sahara Marocain (Tan-Tan). Journal of Animal \& Plant Sciences 2014, 20, 3171-3193.

52. Jouad, H.; Haloui, M.; Rhiouani, H.; El Hilaly, J.; Eddouks, M. Ethnobotanical survey of medicinal plants used for the treatment of diabetes, cardiac and renal diseases in the North centre region of Morocco (FezBoulemane). Journal of Ethnopharmacology 2001, 77, 175-182, https://doi.org/10.1016/s03788741(01)00289-6.

53. Zeouk, I.; Lalami, A.E.O.; Ezzoubi, Y.; Derraz, K.; Balouiri, M.; Bekhti, K. Cutaneous Leishmaniasis: Medicinal Plants Used in Sefrou City (Center of Morocco), a Focus of Leishmaniasis. Phytothérapie 2018.

54. Lekouch, N.; Sedki, A.; Nejmeddine, A.; Gamon, S. Lead and traditional Moroccan pharmacopoeia. Science of the Total Environment 2001, 280, 39-43, https://doi.org/10.1016/s0048-9697(01)00801-4.

55. Teixidor-Toneu, I.; Martin, G.J.; Ouhammou, A.; Puri, R.K.; Hawkins, J.A. An ethnomedicinal survey of a Tashelhit-speaking community in the High Atlas, Morocco. Journal of ethnopharmacology 2019, 188, 96110, https://doi.org/10.1016/j.jep.2016.05.009.

56. Salhi, N.; Bouyahya, A.; Fettach, S.; Zellou, A.; Cherrah, Y. Ethnopharmacological study of medicinal plants used in the treatment of skin burns in occidental Morocco (area of Rabat). South African journal of botany 2019, 121, 128-142, https://doi.org/10.1016/j.sajb.2018.10.038.

57. Tahraoui, A.; El-Hilaly, J.; Israili, Z.H.; Lyoussi, B. Ethnopharmacological survey of plants used in the traditional treatment of hypertension and diabetes in south-eastern Morocco (Errachidia province). Journal of ethnopharmacology 2007, 110, 105-117, https://doi.org/10.1016/j.jep.2006.09.011.

58. Ziyyat, A.; Legssyer, A.; Mekhfi, H.; Dassouli, A.; Serhrouchni, M.; Benjelloun, W. Phytotherapy of hypertension and diabetes in oriental Morocco. Journal of ethnopharmacology 1997, 58, 45-54, https://doi.org/10.1016/s0378-8741(97)00077-9.

59. Bouyahya, A.; Et-Touys, A.; Dakka, N.; Fellah, H.; Abrini, J.; Bakri, Y. Antileishmanial potential of medicinal plant extracts from the North-West of Morocco. Beni-Suef University Journal of Basic and Applied Sciences 2018, 7, 50-54, https://doi.org/10.1016/j.bjbas.2017.06.003.

60. Et-Touys, A.; Fellah, H.; Sebti, F.; Mniouil, M.; Elboury, H.; Talbaoui, A.; Bakri, Y. In vitro antileishmanial activity of extracts from endemic Moroccan medicinal plant Salvia verbenaca (L.) Briq. sspverbenaca Maire (S. clandestina Batt. non L). European Journal of Medicinal Plants 2016, 1-8, https://doi.org/10.9734/EJMP/2016/27891.

61. Et-Touys, A.; Fellah, H.; Mniouil, M.; Bouyahya, A.; Dakka, N.; Abdennebi, E.H.; Bakri, Y. Screening of antioxidant, antibacterial and antileishmanial activities of Salvia officinalis L. extracts from Morocco. $\mathrm{Br}$ MicrobiolRes J 2016, 16, 1-10, https://doi.org/10.9734/BMRJ/2016/28307.

62. Bouyahya, A.; Bakri, Y.; Belmehdi, O.; Et-Touys, A.; Abrini, J.; Dakka, N. Phenolic extracts of Centaurium erythraea with novel antiradical, antibacterial and antileishmanial activities. Asian Pac J Trop Dis 2017, 7, 433-9, https://doi.org/10.12980/apjtd.7.2017D6-462.

63. Bouyahya, A.; Dakka, N.; Talbaoui, A.; Et-Touys, A.; El-Boury, H.; Abrini, J.; Bakri, Y. Correlation between phenological changes, chemical composition and biological activities of the essential oil from Moroccan endemic Oregano (Origanum compactum Benth). Industrial crops and products 2017, 108, 729737, https://doi.org/10.1016/j.indcrop.2017.07.033.

64. Bouyahya, A.; Assemian, I.C.C.; Mouzount, H.; Bourais, I.; Et-Touys, A.; Fellah, H.; Bakri, Y. Could volatile compounds from leaves and fruits of Pistacialentiscus constitute a novel source of anticancer, antioxidant, antiparasitic and antibacterial drugs? Industrial crops and products 2019, 128, 62-69, https://doi.org/10.1016/j.indcrop.2018.11.001.

65. Bouyahya, A.; Et-Touys, A.; Bakri, Y.; Talbaui, A.; Fellah, H.; Abrini, J.; Dakka, N. Chemical composition of Mentha pulegium and Rosmarinus officinalis essential oils and their antileishmanial, antibacterial and antioxidant activities. Microbial pathogenesis $2017, \quad$ 111, https://doi.org/10.1016/j.micpath.2017.08.015.

66. Bouyahya, A.; Et-Touys, A.; Abrini, J.; Talbaoui, A.; Fellah, H.; Bakri, Y.; Dakka, N. Lavandula stoechas essential oil from Morocco as novel source of antileishmanial, antibacterial and antioxidant activities. Biocatalysis and Agricultural Biotechnology 2017, 12, 179-184, https://doi.org/10.1016/j.bcab.2017.10.003.

67. Chalchat, P.; Colas-Belcour, J.; Destombes, P.; Drouhet, E.; Fromentin, H.; Martin, L.; Silverie, J. A Case of Mucocufaneous Leishmaniasis resistant to Antimony Compounds and cured by Amphotericin. Bulletin de la Société de Pathologie Exotique 1965, 58, 73-80. 
68. Medda, S.; Mukhopadhyay, S.; Basu, M.K. Evaluation of the in vivo activity and toxicity of amarogentin, an antileishmanial agent, in both liposomal and niosomal forms. Journal of Antimicrobial chemotherapy 1999, 44, 791-794, https://doi.org/10.1093/jac/44.6.791.

69. Moideen, S.V.L.; Houghton, P.J.; Croft, S.L. Antileishmanial activity of Kigeliapinnata root bark constituents. J. Pharm. Pharmacol 1997, 49.

70. De Carvalho, P.B.; Arribas, M.D.G.; Ferreira, E.I. Leishmaniasis. What do we know about its chemotherapy? Revista Brasileira de Ciências Farmacêuticas 2005, 36, 69-96.

71. Arruda, D.C.; Miguel, D.C.; Yokoyama-Yasunaka, J.K.; Katzin, A.M.; Uliana, S.R. Inhibitory activity of limonene against Leishmania parasites in vitro and in vivo. Biomedicine \& Pharmacotherapy 2005, 63, 643649, https://doi.org/10.1016/j.biopha.2009.02.004.

72. Robledo, S.; Osorio, E.; Munoz, D.; Jaramillo, L.M.; Restrepo, A.; Arango, G.; Vélez, I. In vitro and in vivo cytotoxicities and antileishmanial activities of thymol and hemisynthetic derivatives. Antimicrobial agents and chemotherapy 2005, 49, 1652-1655, https://doi.org/10.1128/aac.49.4.1652-1655.2005.

73. de Morais, S.M.; Vila-Nova, N.S.; Bevilaqua, C.M.L.; Rondon, F.C.; Lobo, C.H.; Moura, A.D.A.A.N.; Wilson, M.E. Thymol and eugenol derivatives as potential antileishmanial agents. Bioorganic \& medicinal chemistry 2014, 22, 6250-6255, https://doi.org/10.1016/j.bmc.2014.08.020.

74. Farias-Junior, P.A.; Rios, M.C.; Moura, T.A.; Almeida, R.P.; Alves, P.B.; Blank, A.F.; Fernandes, R.P.; Scher, R. Leishmanicidal activity of carvacrol-rich essential oil from Lippia sidoides Cham. Biological Research 2012, 45, 399-402, https://doi.org/10.4067/s0716-97602012000400012.

75. Chen, W.; Vermaak, I.; Viljoen, A. Camphor-a fumigant during the black death and a coveted fragrant wood in ancient Egypt and Babylon-a review. Molecules 2013, 18, 5434-5454, https://doi.org/10.3390/molecules18055434.

76. Essid, R.; Rahali, F.Z.; Msaada, K.; Sghair, I.; Hammami, M.; Bouratbine, A.; Limam, F. Antileishmanial and cytotoxic potential of essential oils from medicinal plants in Northern Tunisia. Industrial Crops and Products 2015, 77, 795-802, https://doi.org/10.1016/j.indcrop.2015.09.049.

77. de Lima, S.C.G.; Teixeira, M.J.; Lopes Júnior, J.E.G.; de Morais, S.M.; Torres, A.F.; Braga, M.A.; NagaoDias, A.T. In vitro and in vivo leishmanicidal activity of Astroniumfraxinifolium (Schott) and Plectranthusamboinicus (Lour.) Spreng against Leishmania (Viannia) braziliensis. BioMed research international 2014, 2014, https://doi.org/10.1155/2014/848293.

78. Machado, M.; Dinis, A.M.; Santos-Rosa, M.; Alves, V.; Salgueiro, L.; Cavaleiro, C.; Sousa, M.C. Activity of Thymus capitellatus volatile extract, 1, 8-cineole and borneol against Leishmania species. Veterinary parasitology 2000, 200, 39-49, https://doi.org/10.1016/j.vetpar.2013.11.016. 